\title{
REASSESSING RELIABILITY BASED ON SURVIVED LOADS
}

\author{
Timo Schweckendiek ${ }^{1}$
}

\begin{abstract}
The reliability of flood defenses is often dictated by large uncertainties in the hydraulic loading and the structural resistance. Additional information decreases uncertainty, however, acquiring it is often costly. One source of information, even though in many cases readily available, is hardly used - survived loads. This article shows how data on survived load conditions can be incorporated in reliability analysis by means of Bayesian techniques. The theory is illustrated by simple and realistic examples. In contrast to other sources of information, reassessing structures using survived load data always leads to higher reliability or lower probability of failure. Furthermore, attention is given to the expected development of failure in time. This may be relevant for situations, where the safety requirements of a structure are stated in terms of a design or inspection period. For both, re-assessing reliability based on (one time) survived loads as well as the expected increase of reliability in time, the examples show significant impact. Use of this knowledge may, consequently, safe cost of construction or reinforcement.
\end{abstract}

Keywords: flood defenses; reliability; Bayesian updating; failure mechanisms; piping; survived loads

\section{INTRODUCTION}

Breaching of flood defenses such as dikes can lead to severe inundations and substantial damage in the hinterland. Therefore, not only the height of a dike is important but also its structural resistance against other, mainly geotechnical failure mechanisms like slope failure or piping (also called underseepage). As opposed to the easily measurable geometry (including the height), geotechnical resistance properties are hard to determine, resulting in large uncertainties. These uncertainties regarding the properties of the dike body and its foundation often dominate the probability of failure.

Therefore, every bit of information on the reliability of a flood defense is valuable. One source of information is hardly used: survived loadings. Survived loadings are very much like incomplete load tests, except they are neither intentional nor under controlled conditions. However, as long as the relevant parameters were measured, the reliability or probability of failure can be updated using these data.

This paper not only shows how the probability of failure can be updated by applying Bayesian techniques with historical survival data (posterior analysis), it also treats how we can determine the expected increase of reliability in time (pre-posterior analysis). The theory is illustrated by realistic numerical examples of the piping failure mechanism.

The next chapter provides the mathematical background of reliability theory and Bayesian Updating. After that, simple numerical examples provide an idea of the impact of incorporating survival information. Subsequently, realistic numerical examples illustrate the effects that can be expected applying the presented techniques in practice.

\section{(RE)ASSESSING RELIABILITY}

This chapter recapitulates the basic reliability theory necessary for understanding the Bayesian techniques explained subsequently. It ends with considerations on the expected development of the probability of failure in time.

\section{Prior Probability of Failure}

The general expression for the probability of failure is given by:

$$
P_{f}=P(Z(\mathbf{X})<0)=\int f_{\mathbf{X}}(\mathbf{x}) 1[Z(\mathbf{X})<0] d \mathbf{x}
$$

where $Z(\cdot)$ limit state function

$f_{\mathbf{X}}(\mathbf{x})$ probability density function (p.d.f.) of the vector or random variables $\mathbf{X}$

$1[\cdot]$ indicator function

The integral on the right-hand side can, if analytical solution is not tractable, be solved numerically by well-known techniques, such as the First-Order Reliability Method (FORM, Hasofer and Lind (1974)),

\footnotetext{
${ }^{1}$ Department of Hydraulic Engineering, Delft University of Technology, Stevinweg 1, 2628CN Delft, Netherlands
} 
Monte-Carlo simulation (MCS) or Numerical Integration (NI). The analyses in this paper mainly rely on numerical methods.

If carried out with prior probability distributions of the random variables, the result may be called prior probability of failure, expressing that no additional information than the initially available has been incorporated yet.

\section{Posterior Probability of Failure}

The posterior (or updated) probability of failure can be determined by applying Bayes' theorem (Bayes (1763)) in basically two different fashions, by updating the basic random variables and repeating the reliability re-determining $P_{f}$, or by directly updating $P_{f}$ making use of the definition of conditional probability and the correlation between the historically observed event and the future event to be assessed.

For updating the random variables, first their parameters are updated using Bayes' theorem:

$$
f_{\Theta}(\theta \mid \epsilon)=k \mathbb{L}(\theta) f_{\Theta}(\theta)
$$

where $\Theta$ vector of distribution parameters of random variables $\mathbf{X}\left(f_{\mathbf{X}}(\mathbf{x} \mid \theta)\right)$

$\epsilon$ evidence (information to be incorporated)

$f_{\Theta}(\theta)$ probability density function of $\Theta$

$\mathbb{L}(\theta)$ likelihood function of the parameters $(P(\epsilon \mid \theta))$

$k$ normalization constant (to ensure proper p.d.f.)

Integrating out the uncertainty in the updated parameters gives the updated distribution of the random variables themselves:

$$
f_{\mathbf{X}}(\mathbf{x} \mid \epsilon)=\int f_{\mathbf{X}}(\mathbf{x} \mid \theta) f_{\Theta}(\theta \mid \epsilon) d \theta
$$

Using this updated distribution of the random variables in Eq. (1), gives the posterior probability of failure. The same is achieved by applying the definition of conditional probability:

$$
P\left(Z(\mathbf{X})<0 \mid Z_{h}\left(\mathbf{X}_{h}\right)>0\right)=\frac{P\left(Z(\mathbf{X})<0 \cap Z_{h}(\mathbf{X})>0\right)}{P\left(Z_{h}(\mathbf{X})>0\right)}
$$

where $Z_{h}(\mathbf{X})>0$ describes the historical survival event, while $Z(\mathbf{X})>0$ still describes the future failure event to be assessed. This implies that $\mathbf{X}$ now needs to include the random variables with the information of the historical event. such as information on the observed (deterministic or uncertain) loading conditions.

Since in most cases analytical solutions of the above are intractable or at least cumbersome, again, techniques like MCS or NI can be applied. Alternatively, first-order system reliability methods as proposed by Hohenbichler and Rackwitz (1983) can be used in combination with FORM and are often more insightful.

Notice that in Eq. (4) the same limit state equation $Z_{h}$ for the survival event does not necessarily need to be the same failure mechanism as $Z$. In fact, any event correlated with the failure event to be predicted provides additional information, be it failure or survival regarding another mechanism or measurements or monitoring or relevant properties. The stronger the correlation, the more significant the impact of updating is. The condition, though, is that the information needs to be expressed as an inequality (i.e., $h(\mathbf{X})<0$, see Ditlevsen (1996)).

\section{Expected Development of Probability of Failure in Time ("Pre-posterior”)}

So far, we have treated updating with information of historical or past events. The information may either be deterministic (e.g., measurements with negligible error) or uncertain, the method can cope with both. But what about the future? We already know that the flood defense will experience loadings in the future, too, and probably also extreme ones. In many cases we even have statistics for these loadings, such as extreme value distributions of the annual maxima of river discharges or water levels. Technically, updating a probability of failure with an uncertain future loading is no different than with an uncertain loading in the past. That enables us to analyze the expected development of the probability of failure in time.

In order to develop the necessary mathematics for this purpose, we first introduce the following shorthand notation for failure and survival events: 


$$
F=\{Z(\mathbf{X})<0\} \quad \Leftrightarrow \quad \bar{F}=\{Z(\mathbf{X})<0\}
$$

Furthermore, a time index is introduced. $F_{i}$ means failure in year $i$, or $Z\left(\mathbf{X}_{i}\right)<0$. The index used for the vector of random variables $\mathbf{X}$ means that, since our state of knowledge of the random variables changes, their probability distribution might differ from year to year. Thus in year $i$ we use the probability distribution that includes all the information included up to year $i-1$.

Consequently, the posterior probability of failure in year $i$, given survival in year $j(j<i)$ may be written as $P\left(F_{i} \mid \bar{F}_{j}\right)$. The expected probability of failure in year $i$ depends on the conditional (updated) probability of failure in that year and the probability of survival in the previous years:

$P\left(F_{i}\right)=P\left(F_{i} \mid \bar{F}_{i-1}\right) \prod_{j=1}^{i-2} P\left(\bar{F}_{j+1} \mid \bar{F}_{j}\right) \cdot P\left(\bar{F}_{1}\right)=P\left(F_{i} \mid \bar{F}_{i-1}\right) \cdot\left(1-P\left(F_{i-1}\right)\right)(6)$

Notice that, even though all past events influence $P\left(F_{i}\right)$, the state of knowledge of year $i-1$ is sufficient to determine $P\left(F_{i}\right)$, a sort of Markov-property that is very practical for computational implementation.

\section{LOAD VS. RESISTANCE (Z=R-S)}

In order to illustrate posterior as well as pre-posterior analysis, we first look at the simple classical, but insightful, example of one load and one resistance variable with the limit state function $Z=R-S$ (i.e., failure is $F=\{R<S\}$ ). In all the subsequent cases, we assume the load $S$ to be i.i.d. in time with p.d.f. $f_{S}(s)$.

\section{General Expressions}

Since our state of knowledge of $R$ changes in time, $R_{i}$ is the random variable representing our state of knowledge in year $i$. Thus, the probability of failure in year $i$ may be written as:

$$
P\left(F_{i}\right)=P\left(R_{i}-S_{i}<0\right)=\int F_{R_{i}}\left(s_{i}\right) f_{S}\left(s_{i}\right) d s_{i}
$$

Let a historical survival event be denoted as $\bar{F}_{j}=R_{j}>\tilde{s}_{j}$ (i.e., non-failure in year $j$ ), in which $\tilde{s}_{j}$ is the observed load. Then, the posterior probability of failure in year $i(i>j)$ may be expressed as:

$$
P\left(F_{i} \mid \bar{F}_{j}\right)=P\left(R_{i}<S_{i} \mid R_{j}>\tilde{s}_{j}\right)=\int F_{R_{i} \mid \bar{F}_{j}}\left(s_{i}\right) f_{S}\left(s_{i}\right) d s_{i}
$$

where $F_{R_{i} \mid \bar{F}_{j}}(s)$ is the updated fragility curve (probability of failure, conditional on the load) curve. For more than one, say $M$ survival events, the expression becomes:

$$
P\left(F_{i} \mid \cap \bar{F}_{j}\right)=P\left(R_{i}<S_{i} \mid \bigcap\left\{R_{j}>\tilde{s}_{j}\right\}\right) \quad j=\mathbb{Z}[1, M]
$$

Similarly, we may evaluate the expected development of the probability of failure in time by considering the effect of the uncertain value of the observed load:

$$
P\left(F_{i} \mid \bar{F}_{i-1}\right)=\int F_{R_{i} \mid \bar{F}_{i-1}}\left(s_{i}\right) f_{S}\left(\tilde{s}_{i-1}\right) f_{S}\left(s_{i}\right) d \tilde{s}_{i-1} d s_{i}
$$

Notice that in Eq. (10), the conditional probability of failure in a year, given survival of the previous year, the uncertainty of the load is integrated out twice, once accounting for the uncertainty in the future loading and once accounting for the uncertain effect of updating with the yet unknown load in year $i$.

Applying the law of total probability we need to multiply still with the probability of survival of year $i$ in order to obtain the "expected" probability of failure in year $i+l$ (compare Eq. (6)). Thus, for a time series of the (expected) probability of failure from year $l$ to $N$, we would need to consider the following integral:

$$
P\left(F_{N} \mid \bigcap_{j} \bar{F}_{j}\right)=\int f_{\mathbf{R}, \mathbf{S}}(\mathbf{r}, \mathbf{s}) 1\left[\left\{R_{N}<S_{N}\right\} \cap\left(\bigcap_{j}\left\{R_{j}>S_{j}\right\}\right)\right] d \mathbf{r} d \mathbf{s} j=[1, N-1](11)
$$


where $f_{\mathbf{R}, \mathbf{S}}(\mathbf{r}, \mathbf{s})$ is the joint p.d.f. of the resistance and load variables $R_{i}$ and $S_{i}$ in years $i=\mathbb{Z}[1, N]$. Notice that not only the resistance properties are mutually dependent in subsequent years, but due to the updating based on survival, also the resistance becomes dependent of the loading in previous years. Solving the integral in Eq. (11) for cases without special (in)dependence assumptions of the resistance in time (i.e., $0<\rho\left(R_{i}, R_{j}\right)<1$ ), is not trivial and left for further research.

\section{Full Correlation of the Resistance in Time}

From this point on, we assume the uncertainty in the resistance to be purely epistemic. In other words, it has a time-invariant but unknown and, thus, uncertain value. Theoretically, this type of uncertainty can be eliminated with sufficient measurements or gathering of information, which, however, is often not possible in practice.

This assumption seems reasonable and is very common for strength properties of defenses, as long as no time-dependent behavior like deterioration is considered. For example, soil properties are realized once during the deposition of the respective strata. They could be known deterministically, if we just carried out enough soil investigation. Nevertheless, we treat them as random variables to cope with our limited knowledge, because "complete" soil investigation is not practicable. Furthermore, from a risk analysis or decision theoretical point of view, there is no difference between "true" inherent variability or randomness and uncertainty arising from lack of knowledge or epistemic uncertainty (Faber et al. (2007)).

Now, since the resistance is a one time-realization, we also need only one random variable $R$ to describe it (i.e., index $i$ is not needed anymore). Notice that this is equivalent to having full correlation of the resistance in time $\left(\rho\left(R_{i}, R_{j}\right)=1\right)$. Furthermore, the effect of updating the resistance with a survived load is, in fact, truncating the distribution. Intuitively, this is easily understood, because, if the resistance has an unknown value and no inherent randomness is involved, this value must surely be greater than the observed load. This is illustrated in Figure 1, where R' represents the posterior distribution of $\mathrm{R}$ and the shaded area shows the redistributed probability mass.

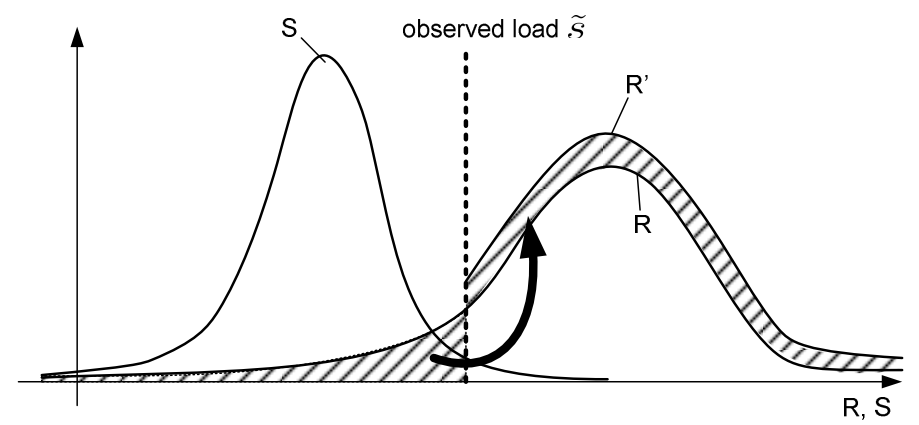

Figure 1: Re-distribution of Probability Mass for Observed Loads and Knowledge Uncertainty in Resistance

Bayesian Theory provides an explanation for this effect, too. The likelihood function of $R$ may be formulated as:

$$
\mathbb{L}(R)=P(\bar{F} \mid \tilde{s})=P(R>\tilde{s})=1[R>\tilde{s}]
$$

Notice that the likelihood function directly works on R, not on parameters of a distribution of $R$, because we assume $R$ to have a real value, not to be a random process. The function assumes value 1 for $\mathrm{R}$ greater than $\tilde{s}$ and 0 elsewhere. Multiplying this function with the prior distribution and normalizing has exactly the effect of truncating the distribution as illustrated in Figure 1.

This effect has another computational advantage. Now, only the maximum observed load contributes to the updating. That implies that for the expected (future) development of $P\left(F_{N}\right)$ considering the (extreme value) distribution of the maximum load per time interval suffices. Consequently, the posterior probability of failure, as compared to equation (9), simplifies to

$$
P\left(F_{i} \mid \cap \bar{F}_{j}\right)=P\left(R<S_{i} \mid \bigcap\left\{R>\max \left[\tilde{s}_{j}\right]\right\}\right)=\int F_{R \mid R>\max \left[\tilde{s}_{j}\right]}\left(s_{i}\right) f_{S}\left(s_{i}\right)(13)
$$

where the notation $F_{R \mid R>\tilde{s}}$ stands for a c.d.f. of R, where the left tail of its distribution is truncated at $\tilde{s}$. Consequently, the expected (future) conditional probability of failure becomes: 


$$
P\left(F_{N} \mid F_{N-1}\right)=\int F_{R \mid R>s}\left(s_{N}\right) f_{S_{N-1}}(s) f_{S}\left(s_{N}\right) d s d s_{N}
$$

where $f_{S_{N-1}}(s)$ is the p.d.f. of the maximum load in $N-1$ years. According to Eq. (6), this conditional probability needs to be multiplied with the probability of survival of the previous year in order to obtain $P\left(F_{N}\right)$. However, if the prior probability of failure is low, the survival terms are close to 1 and $P\left(F_{N}\right)$ can be approximated by Eq. (14) with reasonable accuracy.

\section{EXAMPLES}

\section{Single Parameter Updating (Z=R-S)}

This example shows how for the simple problem of $Z=R-S$ Bayes' theorem can be useful to deal with situations where the uncertainty in the resistance consists of both, lack of knowledge and inherent variability. One possibility of dealing with this is to have a distribution of the mean value $\mu_{R}$ of a Normal-distributed variable $R$ accounting for the reducible part of the uncertainty, while the standard deviation $\sigma_{R}$ is assumed constant representing the non-reducible (i.e., inherent) uncertainty. The properties of our numerical example are:

- $\quad$ prior distribution of the mean: $\mu_{R} \sim N(\mu, \sigma)=N(5,1)$

- $\quad$ standard deviation: $\sigma_{R}=1$

- $\quad$ prior (Bayesian) distribution of the resistance: $R \sim N\left(\mu, \sqrt{\sigma^{2}+\sigma_{R}^{2}}\right)=N(5, \sqrt{2})$

- load distribution: $S \sim N\left(\mu_{S}, \sigma_{S}\right)=N(2,1)$

- $\quad$ prior reliability index and probability of failure:

$$
\beta=\mu_{Z} / \sigma_{Z}=\mu / \sqrt{\sigma^{2}+\sigma_{R}^{2}+\sigma_{S}^{2}}=5 / \sqrt{3}=1.73 \Rightarrow P(F)=\Sigma(-1.73)=4.2 \cdot 10^{-2}
$$

A practical example, where such a distinction between reducible and non-reducible uncertainty may be applicable, is the characterization of a soil property within a site. A typical value for the within-site variability is often known, while for a new, less explored site the mean value is estimated based on a limited number of samples.

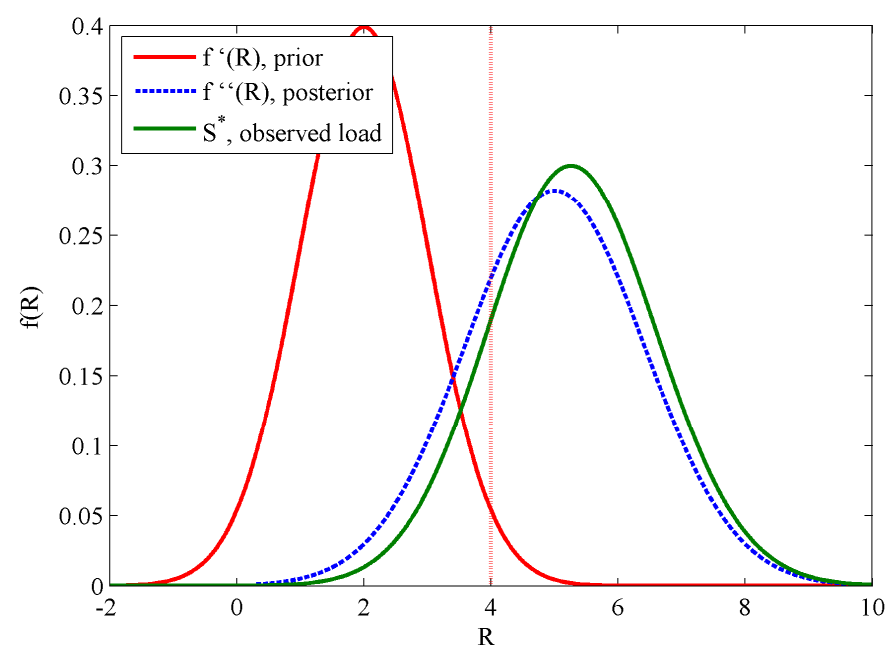

Figure 2: The Effect of Including a Survival Observation in de Resistance Distribution

Figure 2 shows the effect of updating the (mean value) of the resistance based on an observed (survived) load of $\tilde{s}=4$. Clearly, in this case there is no truncating of the prior distribution, rather a shift to the right narrowing of the distribution. The reason is that there is non-reducible uncertainty involved, the result of which is that also our observation is not as sharp as it would be with only lack of knowledge.

The likelihood function of $\mu_{R}$ (i.e., the updated parameter) contains $\sigma_{S}$, the non-reducible part of the uncertainty. Letting its value approach zero (Eq. (15)), illustrates the effect explained above. The distribution is effectively truncated: 
$L\left(\mu_{R}\right)=P\left(R>\tilde{S} \mid \mu_{R}\right)= \begin{cases}\lim _{\sigma_{S} \rightarrow 0} \Phi\left(-\frac{\tilde{s}-\mu_{R}}{\sigma_{S}}\right)=\Phi(-\infty)=0 & \text { if } \mu_{R}<\tilde{s} \\ \lim _{\sigma_{S} \rightarrow 0} \Phi\left(-\frac{\tilde{S}-\mu_{R}}{\sigma_{S}}\right)=\Phi(+\infty)=1 & \text { if } \mu_{R}>\tilde{s}\end{cases}$

Figure 3 illustrates the effect of updating the resistance on the posterior probability of failure depending on the magnitude on the loading event. In terms of probability of failure we are usually interested in changes of orders of magnitude. For this specific example, the observed load would have to be very high to have such an impact. For example, the prior probability of failure for the observed event (Figure 2) was approximately $24 \%$ and the probability of failure only decreased by a factor of two. For lower prior probabilities of failure, the impact is usually higher as we will see in other examples.

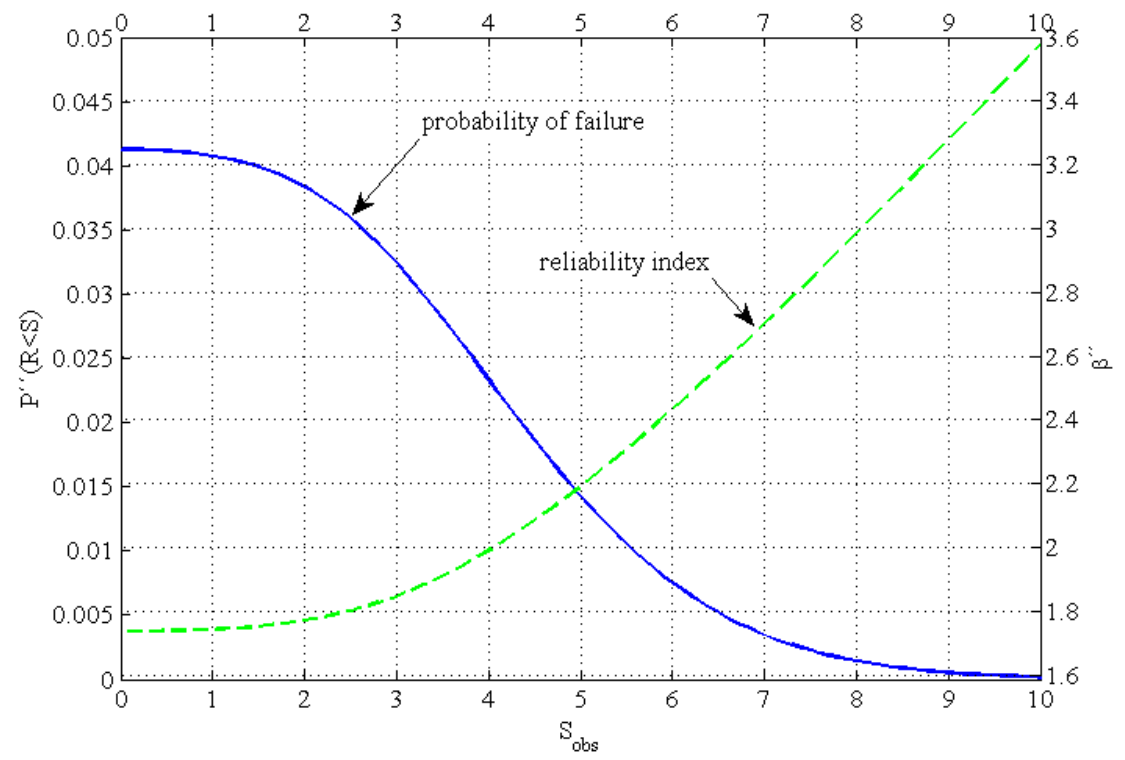

Figure 3: Posterior Probability of Failure as Function of the Observed Survived Load

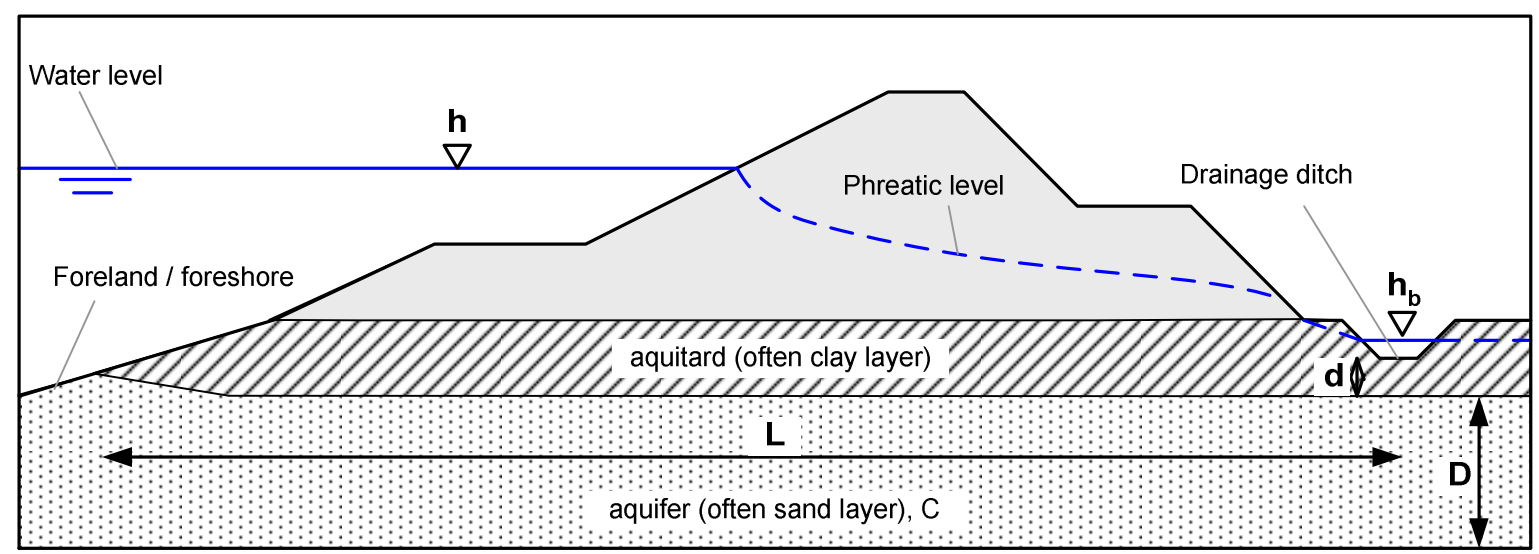

Figure 4: Illustration of schematic geometry and parameters for heave and piping

\section{Multiple Parameter Updating (Piping: Bligh's Rule)}

This example illustrates the effects of updating multiple parameters for the failure mechanism with realistic load and resistance values. To this end the piping model first established by Bligh (1910) is adopted (see Figure 4). The limit state function (LSF) for Bligh's rule is given by:

$$
Z_{p}=L-m_{C} C\left(h-h_{b}-0.3 d\right)
$$


with $\quad L \quad$ piping length $[\mathrm{m}]$

$C \quad$ Bligh's Creep parameter [-] (erosion resistance)

$m_{C} \quad$ factor for model uncertainty associated with $C$

$h \quad$ water level (main load) [m+NAP]

$h_{b} \quad$ landside water level [m+NAP]

d. thickness of aquitard at exit point $[\mathrm{m}]$

The formulation is similar to Steenbergen and Vrouwenvelder (2003), re-written in a linear form. The following assumptions hold:

- If the maximum head difference exceeds the critical head difference, failure occurs (i.e., timedependent effects in the piping mechanism are disregarded).

- No cumulative effect, i.e. there is no cumulative damage or "fatigue" effect.

- Piping can occur directly, i.e. there is no dependence on other mechanisms such as uplift. This assumption is realistic in the absence of blanket layers or for thin blanket layers with very low uplift resistance.

The modeling of uncertainties in piping resistance for this study is adopted from the FLORIS project (VNK) in the Netherlands (Rijkswaterstaat (2005)), the distribution parameters are summarized in Table 1.

\begin{tabular}{|c|c|c|c|c|}
\hline \multicolumn{5}{|c|}{$\begin{array}{l}\text { Table 1: Prior distributions (Piping: Bligh's Rule) } \\
\begin{array}{l|l|l}(\mathrm{NAP}=\text { reference level: Nieuw Amsterdam Peil (mean sea level)) } \\
\text { Variable } & \text { Distribution } & \text { Parameters / moments }\end{array}\end{array}$} \\
\hline$C$ & {$[-]$} & Lognormal & $\mu_{C}=15.0$ & $\sigma_{C}=2.0$ \\
\hline$m_{C}$ & {$[-]$} & Normal & $\mu_{m_{C}}=1.0$ & $\sigma_{m_{C}}=0.15$ \\
\hline & {$[\mathrm{m}]$} & Normal & $\mu_{L}=30.0$ & $\sigma_{L}=3.0$ \\
\hline & [m+NAP] & Gumbel & \multicolumn{2}{|l|}{$\alpha=8.27$} \\
\hline & [m+NAP] & deterministic & \multicolumn{2}{|l|}{$h_{b}=7.5$} \\
\hline & {$[\mathrm{m}]$} & deterministic & \multicolumn{2}{|l|}{$d=0.8$} \\
\hline
\end{tabular}

In the subsequent examples it is assumed that a water level with a 100 year return period of $\tilde{h}=10.14$ [m+NAP] has been observed and no piping failure has occurred.

For sake of illustration, we consider different cases in terms of updating uncertainties. The first case is artificially simplified, only $C$ is considered uncertain on the resistance side, $L$ and $m_{c}$ are considered deterministic with their respective mean values. In order to be able to compare the cases, the standard deviation of $C$ is chosen as $\sigma_{c}=3.0$, which can be shown is approximately equivalent to the combined distribution of $m_{c}{ }^{*} C$ with their original parameters from Table 1 . The uncertainty in $C$ is assumed to be reducible. The likelihood function of $C$ can be formulated as:

$$
\mathbb{L}(C)=P\left(L-m_{C} C\left(\tilde{h}-h_{b}-0.3 d\right)>0 \mid C\right)
$$

$C$ being the only random variable, the likelihood function is deterministic may be re-written as:

$$
\mathbb{L}(C)= \begin{cases}P\left(Z_{p}>0 \mid C\right)=1 & \text { if } C<C_{c} \\ P\left(Z_{p}>0 \mid C\right)=0 & \text { if } C \geq C_{c}\end{cases}
$$

with $\quad C_{c}=L /\left(\tilde{h}-h_{b}-0.3 * d\right)=$ critical Bligh parameter [-]

In other words, the survival of $\tilde{h}$ has proven that $C$ must be smaller than $C_{c}$. Figure 5 shows the joint p.d.f. and the limit state $\left(\mathrm{Z}_{\mathrm{p}}=0\right)$ in h-C-space. The probability mass above the $C_{c}$-line needs to be redistributed below that line $\left(C_{c}<12.51\right)$.

Figure 6 shows the (truncated) posterior distribution of $\mathrm{C}$ and the updated fragility curve $\left(P\left\{Z_{p}<0 \mid h\right\}\right)$. 


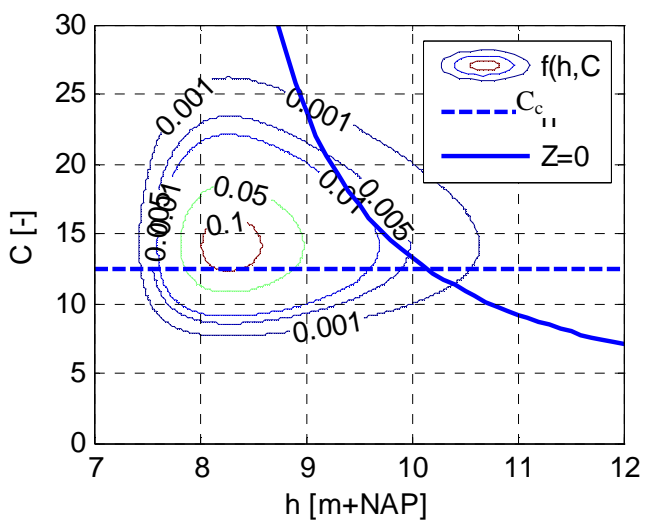

Figure 5: Joint p.d.f. and Limit state in h-C-Space (Piping: Bligh's Rule, only C uncertain)

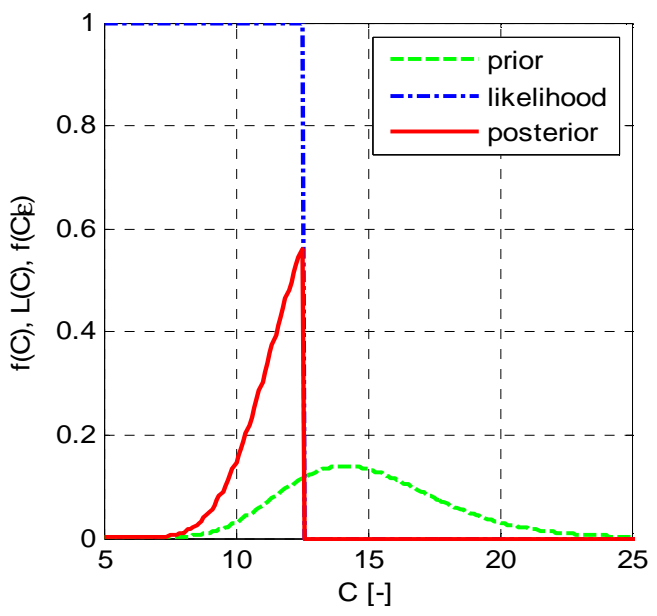

(a) Prior and posterior distribution of $\mathrm{C}$

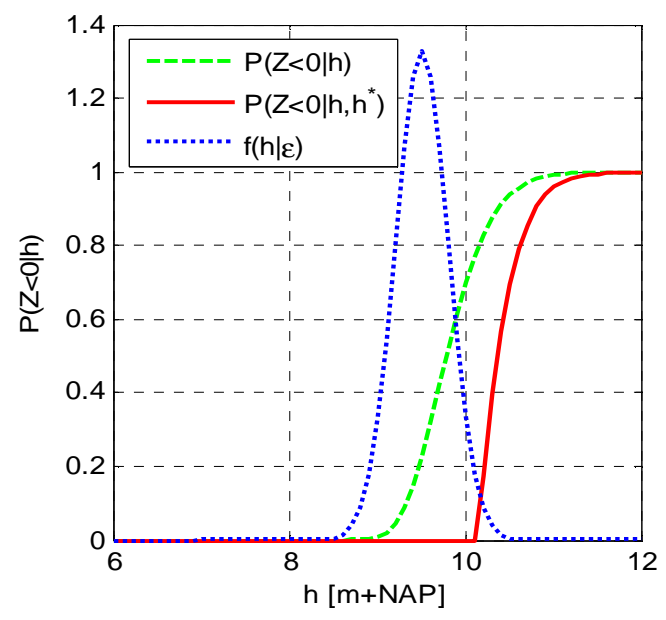

(b) Prior and posterior fragility curves

Figure 6: Updated p.d.f. of $C$ and Updated Fragility Curve (Piping: Bligh's Rule, only $C$ uncertain)

Figure 6 shows the (truncated) posterior distribution of $\mathrm{C}$ and the updated fragility curve $\left(P\left\{Z_{p}<0 \mid h\right\}\right)$, together with an example of a short term water level prediction with expectation $E[h \mid \varepsilon]=9.5$ [m+NAP] and standard prediction error of $\sigma_{h \mid \epsilon}=0.3[\mathrm{~m}]$ (typical for about 3 days lead time on rivers) in order to appreciate the impact of the updating procedure on short-term predictions.

The probability of failure, using the water level distribution of the yearly maxima (long-term), decreases by a factor of 5 . The effect on the short-term reliability with the scenario of a water level prediction as in Figure 6 is even more significant.

\begin{tabular}{|c|c|c|c|}
\hline & & with $\mathrm{f}(\mathrm{C})$ & with $\mathrm{f}(\mathrm{C} \mid \varepsilon)$ \\
\hline \multirow[t]{2}{*}{ with $f(h)$ (long-term) } & Probability of failure & $3.1 \mathrm{E}-2$ & $6.1 \mathrm{E}-3$ \\
\hline & Reliability index & 1.86 & 2.51 \\
\hline \multirow[t]{2}{*}{ with $f(h / \varepsilon)$ (short-term) } & Probability of failure & $2.8 \mathrm{E}-1$ & $4.1 \mathrm{E}-3$ \\
\hline & Reliability index & 0.59 & 2.63 \\
\hline
\end{tabular}

In the second case, model uncertainty is added as non-reducible uncertainty, whilst the piping length $L$ is still treated deterministically. $C$ still being the only random variable, the likelihood function remains the same as Eq. (17). However, in this case, it is a stochastic function, since $m_{c}$ is uncertain. Figure 7 shows that the effect of updating is less than in the previous case, since no truncating occurs anymore. That is also expressed in the impact on the probability of failure being much less. The same holds for the fragility curve and the short-term reliability (see Table 3). 


\begin{tabular}{|c|c|c|c|}
\hline & & with $\mathrm{f}(\mathrm{C})$ & with $\mathrm{f}(\mathrm{C} \mid \varepsilon)$ \\
\hline \multirow[t]{2}{*}{ with $f(h)$ (long-term) } & Probability of failure & $3.1 \mathrm{E}-2$ & $1.8 \mathrm{E}-2$ \\
\hline & Reliability index & 1.86 & 2.09 \\
\hline \multirow[t]{2}{*}{ with $f(h / \varepsilon)$ (short-term) } & Probability of failure & $2.8 \mathrm{E}-1$ & $1.4 \mathrm{E}-1$ \\
\hline & Reliability index & 0.59 & 1.08 \\
\hline
\end{tabular}

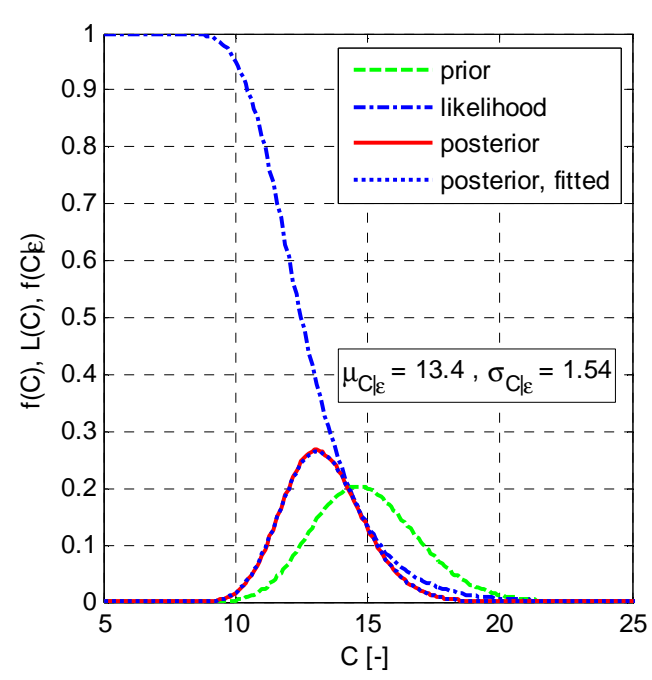

(a) Prior and posterior distribution of $\mathrm{C}$ (short-term)

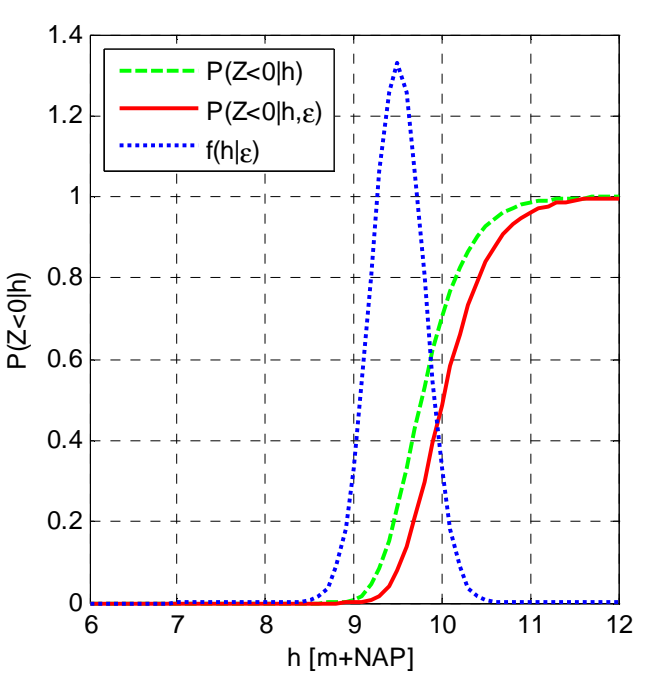

(b) Prior and posterior fragility curves (long-term)

Figure 7: Updated p.d.f. of $\mathrm{C}$ and Updated Fragility Curve (Piping: Bligh's Rule, $\mathrm{C}$ and $\mathrm{m}_{\mathrm{c}}$ uncertain)

In the third case, $C$ and $L$ are considered uncertain, whilst model uncertainty is disregarded $\left(m_{c}=1\right)$. The aggregate uncertainty in $C$ and $L$, is comparable to the total resistance uncertainty in the previous cases. Furthermore it is considered reducible. Consequently, the likelihood function is two-dimensional:

$$
\mathbb{L}\left((C, L)=P\left(L-m_{C} C\left(\tilde{h}-h_{b}-0.3 d\right)>0 \mid C, L\right)\right.
$$

Without (inherent) model uncertainty, the likelihood function is again deterministic, defining an "impossible" parameter region. Figure 8 illustrates the truncating of the joint p.d.f..

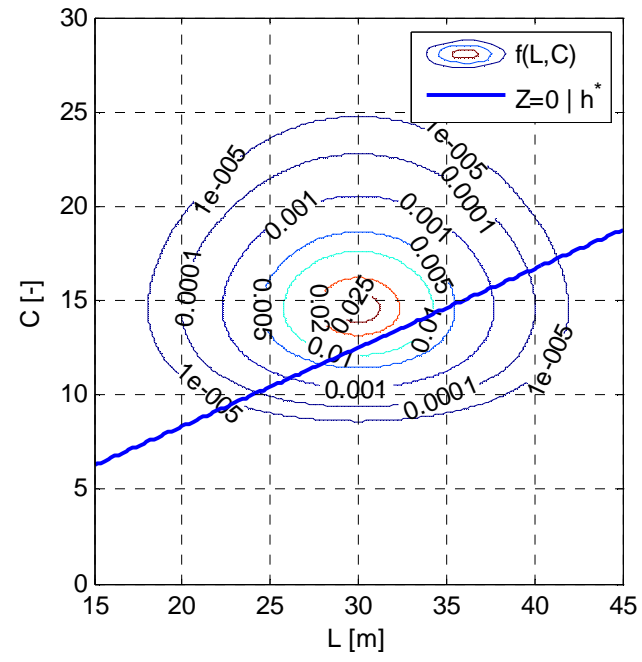

(a) Prior joint p.d.f.

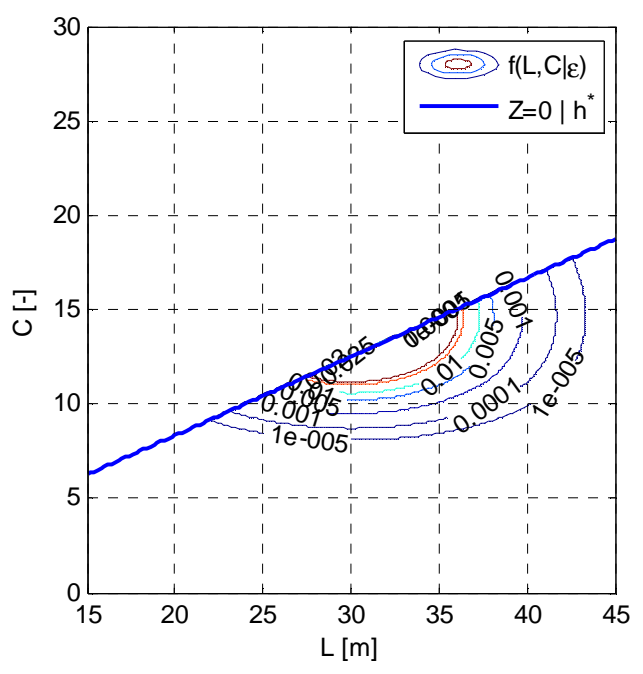

(b) Posterior joint p.d.f.

Figure 8: Updated joint p.d.f. of $C$ and $L$ (Piping: Bligh's Rule, $C$ and $L$ uncertain) 
The posterior marginal distributions (in general: $f_{X}(x)=\int_{y} f_{X, Y}(x, y) d y$ ), of $C$ and $L$ differ significantly from the prior ones (see Figure 9). The posterior marginal distributions are not independent as opposed to the prior distributions. Thus either the full joint posterior p.d.f. needs to be used in subsequent analyses or an appropriate representation of the correlation structure.

In the current analysis, the posterior reliability analyses have been carried out using numerical integration of the joint p.d.f. $f(C, L \mid \epsilon)$ (fourth column in Table 4). In order to demonstrate the error using independent marginal distributions, these results are given in the last column of the same table $(f(C \mid \epsilon) f(L \mid \epsilon))$.

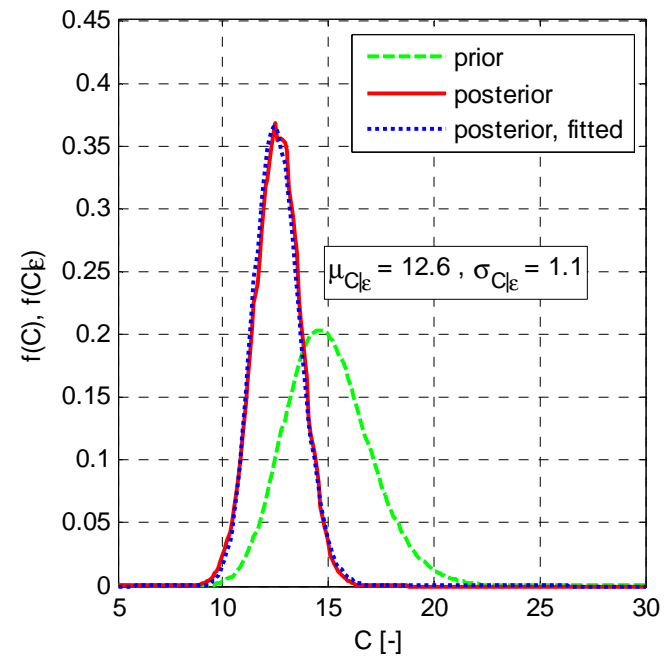

(a) $f(C \mid \epsilon)$

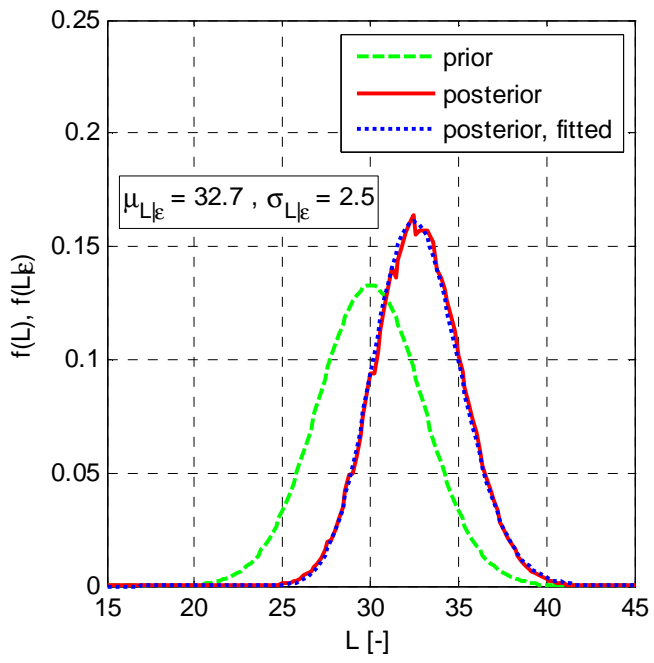

(b) $f(L \mid \epsilon)$

Figure 9: Posterior Marginal Distributions of $C$ and L (Piping: Bligh's Rule, $C$ and $L$ uncertain)

It is remarkable that the posterior probabilities of failure, i.e. the effects of updating the resistance variables, are practically the same as in case 1 (only $\mathrm{C}$ uncertain). Apparently, for the same amount of aggregate uncertainty it is the degree of reducible uncertainty involved that determines the effect on the probability of failure. The same holds for the posterior fragility curve and the effect on the short-term reliability.

\begin{tabular}{|c|c|c|c|c|}
\hline & with $f(C)$ & with $\mathrm{f}(\mathrm{C} \mid \varepsilon)$ & with $\mathrm{f}(\mathrm{C} \mid \varepsilon)$ & with $\mathrm{f}(\mathrm{C} \mid \varepsilon) \mathrm{f}(\mathrm{L} \mid \varepsilon)$ \\
\hline \multirow[t]{2}{*}{ with $f(h / \varepsilon)$ (long-term) } & Probability of failure & $3.1 \mathrm{E}-2$ & $6.4 \mathrm{E}-3$ & $7.5 \mathrm{E}-3$ \\
\hline & Reliability index & 1.86 & 2.49 & 2.43 \\
\hline \multirow[t]{2}{*}{ with $f(h / \varepsilon)$ (short-term) } & Probability of failure & $2.8 \mathrm{E}-1$ & $5.5 \mathrm{E}-3$ & \\
\hline & Reliability index & 0.59 & 2.54 & \\
\hline
\end{tabular}

In the last case, all random variables are treated as uncertain (specifications in Table 1). Notice that the total uncertainty is different and that the results cannot be compared to the previous cases directly anymore. The likelihood function is the same as in the previous case (Eq. (19)), however, in this case it is a stochastic function. In the previous case, the likelihood function distinguished sharply between zero and one probability, in the current case it is a smooth function (see Figure 10).

The posterior marginal distributions and their statistical moments are displayed in Figure 11. Neither the shift in mean value nor the decrease in variance is as significant as in the previous case. The model uncertainty weakens the updating effect, as already shown in the second case.

The effect on the probability of failure (see Table 5) and the fragility curve is similar to the second case, even though the two cases are not perfectly comparable anymore due to the difference in total (aggregate) prior uncertainty. 


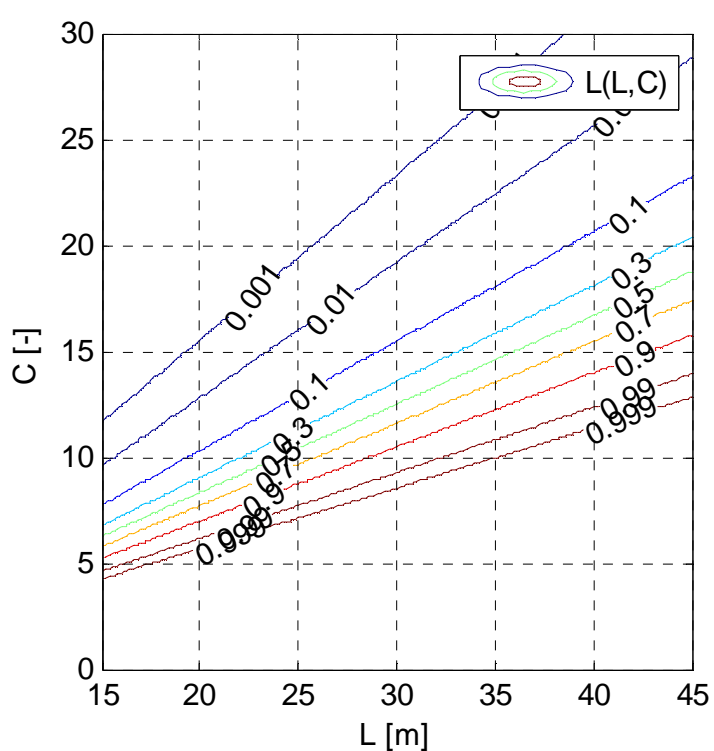

(a) Likelihood

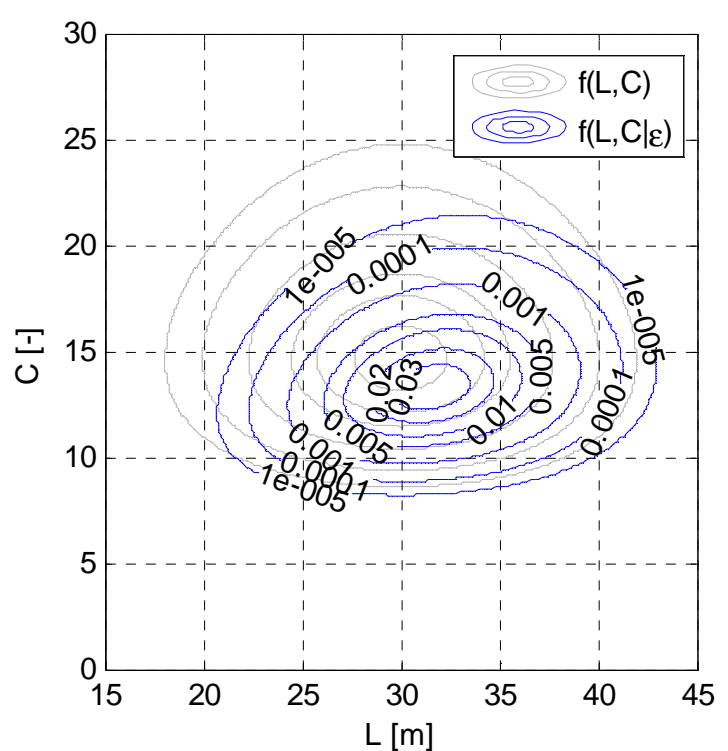

(b) Posterior joint p.d.f.

Figure 10: Contours of the Likelihood and Posterior p.d.f. of $C$ and $L$ (Piping: Bligh's Rule, all variables uncertain)

\begin{tabular}{|c|c|c|c|}
\hline & & with $\mathrm{f}(\mathrm{C})$ & with $\mathrm{f}(\mathrm{C} \mid \varepsilon)$ \\
\hline \multirow[t]{2}{*}{ with $f(h)$ (long-term) } & Probability of failure & $3.7 \mathrm{E}-2$ & $1.6 \mathrm{E}-2$ \\
\hline & Reliability index & 1.79 & 2.13 \\
\hline \multirow[t]{2}{*}{ with $f(h / \mathcal{E})$ (short-term) } & Probability of failure & $2.9 \mathrm{E}-1$ & $1.1 \mathrm{E}-1$ \\
\hline & Reliability index & 0.54 & 1.20 \\
\hline
\end{tabular}

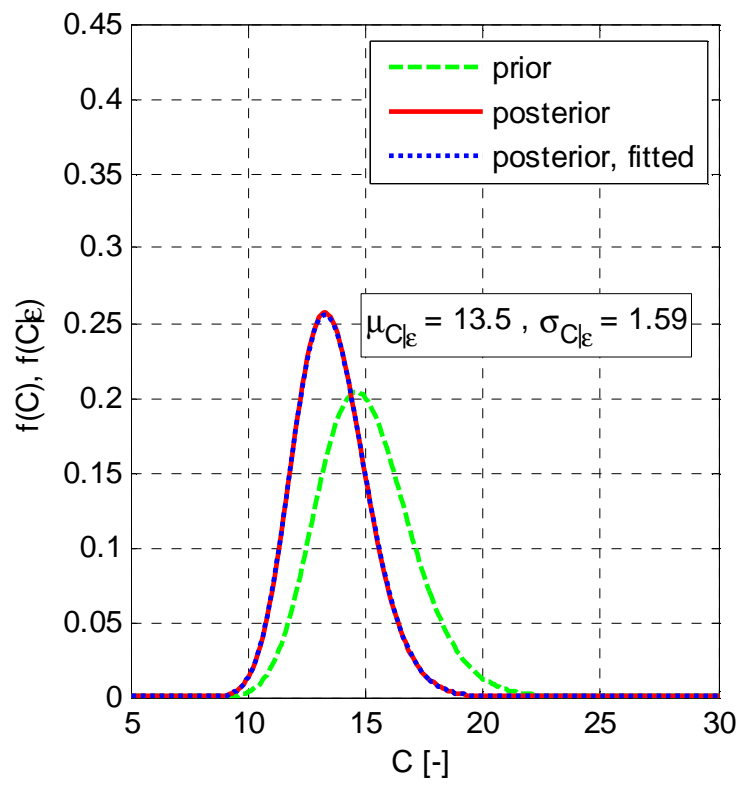

(a) $f(C \mid \epsilon)$

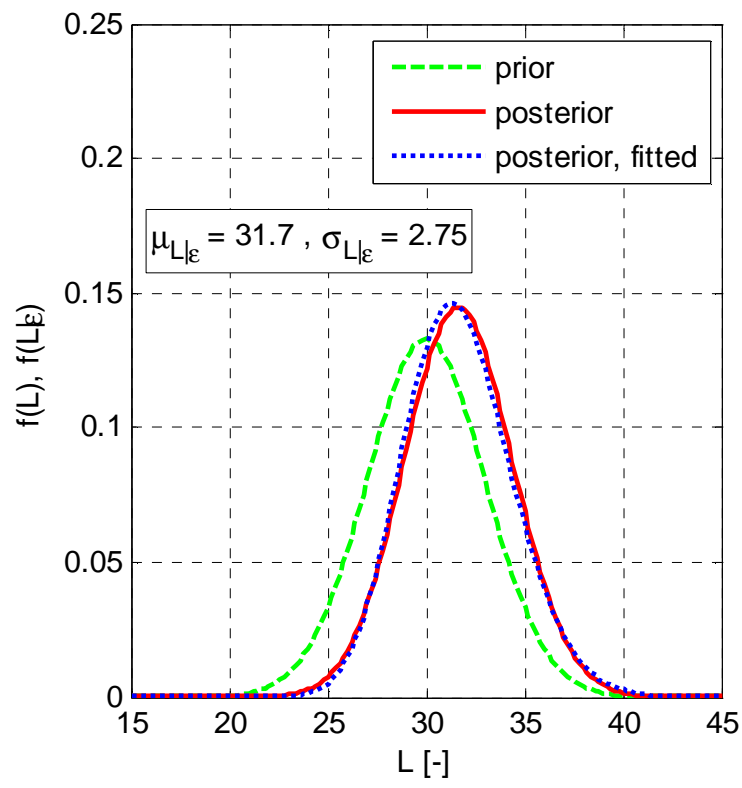

(b) $f(L \mid \epsilon)$

Figure 11: Posterior Marginal Distributions of $C$ and L (Piping: Bligh's Rule, all variables uncertain) 
Time Series (Piping, simplified: $\mathrm{Z}_{\mathrm{p}}=\mathrm{H}_{\mathrm{c}}-\mathrm{H}$ )

This example is concerns the effect of expected survival of future loadings on the probability of failure. Again piping (under-seepage) is used as an example. A simplified piping model is adopted, in which all resistance-related uncertainties are aggregated in the critical head difference $H_{c}$ and the loadrelated uncertainties in the predicted head difference $H$. The distributions and parameters in this example have been derived from analyses with the more sophisticated Sellmeijer (1988) formula, a model physical process-based model for determining the critical head difference.

The main uncertainty in the load stems from the water level, the extreme value distribution of which is commonly described by a Gumbel distribution (for yearly maxima):

$$
F_{H}(h)=\exp \left[-\exp \left(-\frac{h-\alpha}{\beta}\right)\right]
$$

Using the fact that $N=\exp (\ln (N))$, the distribution of the maximum in $N$ years can be written as:

$F_{H_{N}}\left(h_{N}\right)=\left\{\exp \left[-\exp \left(-\frac{h-\alpha}{\beta}\right)\right]\right\}^{N}=\exp \left[-\exp \left(-\frac{h-\alpha+\beta \ln (N)}{\beta}\right)\right](21)$

Thus, $F_{H_{N}}\left(h_{N}\right)$ is also Gumbel-distributed with parameters $\alpha_{N}=\alpha+\beta \ln (N)$ and $\beta_{N}=\beta$. In this example, the Gumbel distribution is not only applied for the water level but also for the head difference $H=h-h_{b}$. Table 6 provides three realistic parameter sets for piping $\mathrm{s}$ in the Netherlands derived from location-specific design water levels (MHW), which correspond with region-specific protection levels expressed in terms of exceedance probabilities ( $F_{\text {exc }}$, see Rijkswaterstaat $\left.(2007)\right)$, combined with the so-called decimate height $h_{\text {dec }}$ (Rijkswaterstaat (2008)).

\begin{tabular}{|c|c|c|r|r|r|r|r|}
\hline \multicolumn{6}{|c|}{ Table 6: Typical Hydraulic Load Conditions for Piping in the Netherlands (normalized to mean sea level) } \\
\hline Location & \multicolumn{1}{|c|}{ MHW- $\mathbf{h}_{\mathbf{b}}$} & $\begin{array}{c}\mathbf{1} / \mathbf{F}_{\text {exc }} \\
{[\mathbf{1} / \mathbf{y r}]}\end{array}$ & $\begin{array}{c}\mathbf{h}_{\text {dec }} \\
{[\mathbf{m}]}\end{array}$ & $\boldsymbol{\beta}$ & $\boldsymbol{\alpha}$ & $\boldsymbol{\alpha}_{\mathbf{5}}$ & $\boldsymbol{\alpha}_{\mathbf{5 0}}$ \\
\hline $\begin{array}{c}\text { Rivers } \\
\text { (upstream) }\end{array}$ & 3.0 & 1,250 & 0.70 & 0.304 & 0.83 & 1.05 & 1.35 \\
\hline $\begin{array}{c}\text { Rivers } \\
\text { (downstream) }\end{array}$ & 5.0 & 4,000 & 0.35 & 0.152 & 3.74 & 3.85 & 4.00 \\
\hline $\begin{array}{c}\text { Coast, } \\
\text { estuaries }\end{array}$ & 5.5 & 10,000 & 0.75 & 0.326 & 2.50 & 2.73 & 3.05 \\
\hline
\end{tabular}

The (prior) resistance is assumed Normal-distributed (Gaussian) with a coefficient of variation of about $20 \%$ and reducible (i.e., lack of knowledge only). Consequently, the updated distribution is a Truncated Normal distribution with the maximum observed load $\tilde{h}_{N}$ as lower bound (see Eq. ).

$$
f_{H_{c}}\left(h_{c} \mid h_{c}>\tilde{h}_{N}\right)=\frac{1}{\sigma_{H_{c}}} \phi\left[\frac{h_{c}-\mu_{H_{c}}}{\sigma_{H_{c}}}\right] /\left(1-\Phi\left[\frac{\tilde{h}_{N}-\mu_{H_{c}}}{\sigma_{H_{c}}}\right]\right)
$$

In the absence of an analytical expression we denote the cumulative distribution as $F_{H_{c}}\left(h_{c} \mid h_{c}>\tilde{h}_{N}\right)$. For the present example, the mean values of the critical head difference per load parameter set (Table 6) have been chosen such, that the prior probability of failure equals the exceedance frequency $\left(P\left(F_{1}\right)=F_{\text {exc }}\right)$. Figure 12 shows the expected development of the probability of failure in time. The difference in decrease of $P\left(F_{i}\right)$ between the different parameter sets is remarkable. It is speculated that the main differences lie in the relative contributions of load and resistance to the total uncertainty and, furthermore, in the level of the probability of failure (i.e., high prior reliability gives high relative decrease). However, a thorough analysis of these differences is beyond the scope of this paper. The decrease in $P\left(F_{i}\right)$ in 50 years is between a factor 2 and 100. Consequently the difference in terms of reliability index is highest for the "Rivers (downstream)" case with about 1.1. 

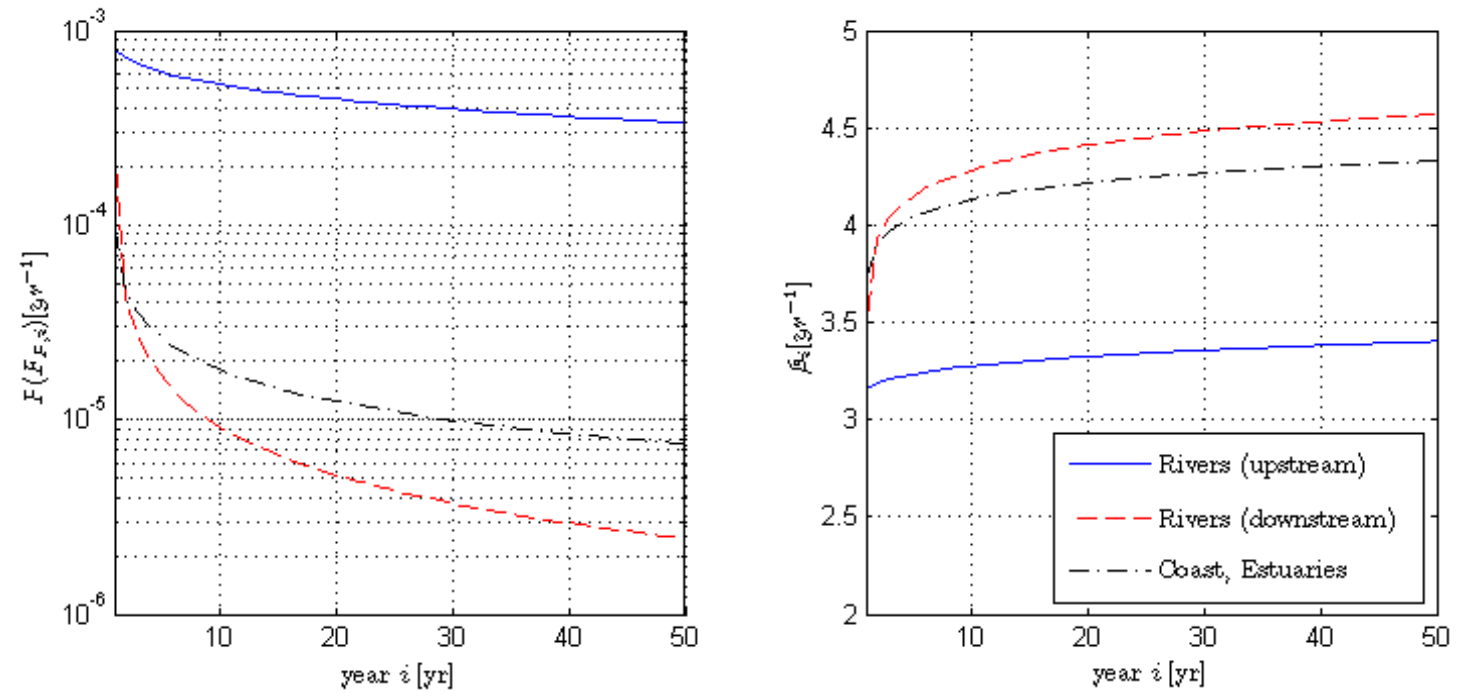

Figure 12: Expected Development of Probability of Failure in Time (Piping, Zp=Hc-H)

Usually, acceptable probabilities of failure in design codes or other requirements to the safety of structures are risk-motivated. An acceptable risk may be derived from cost-benefit considerations, individual or group risk or other criteria. In any case, the derived criterion means that certain damage may only occur with an acceptably low probability. This probability is usually expressed for a reference period of time, often per year or per lifetime. If defined per lifetime or per maintenance or inspection period, the expected decrease of probability of failure in time can be taken into account. In that case, the average of $P\left(F_{i}\right)$ in the reference period is of interest.

Figure 13 shows that the time-averaged probability of failure is considerably lower than the prior. For a typical inspection period of 10 years, the differences are between factor 1.3 and 6 . For a typical design lifetime of 50 years the factor are even roughly between 2 and 20 .
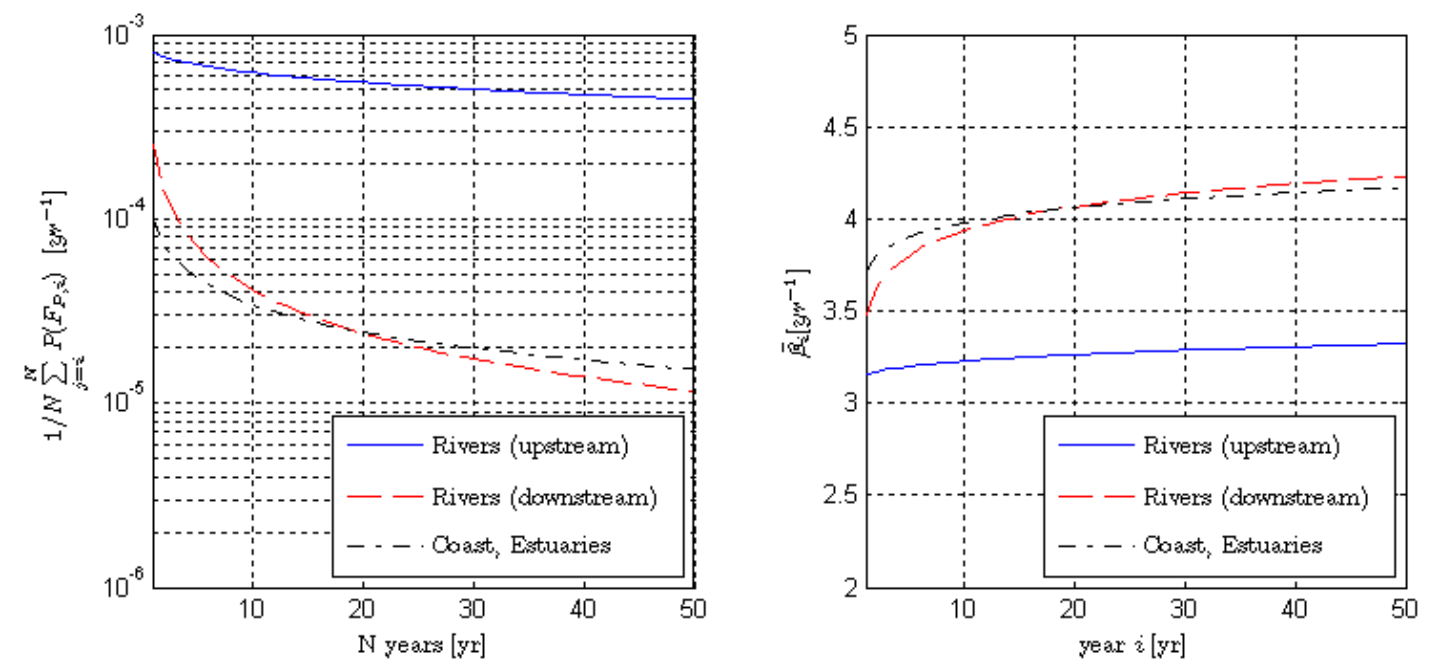

Figure 13: Time-Averaged Expected Probability of Failure in Time (Piping, $\mathrm{Zp}=\mathrm{Hc}-\mathrm{H}$ )

Of course, for using the average value, certain assumptions need to hold, for example, that the damage caused by the unwanted event is insensitive to the event intensity (i.e., the damage by a low-frequency event is not much different to the damage by an event with slightly lower frequency) etc. The analysis above only demonstrates the potential of taken the updating effects into account, for real-life applications, all explicit and implicit assumptions need to be checked carefully. 


\section{DISCUSSION}

The considerations and results provided above have lead to the following conclusions and recommendations:

1. Survived historical loadings are valuable information for the reliability of a flood defense, actually for any kind of structure, especially, if large uncertainties of the resistance dominate the probability of failure. So far, this source of information is hardly used, at least not in a consistent reliability analysis and risk management framework.

2. The present article demonstrates how information on historical and future survived loads can be used to update the probability of failure by means of Bayesian Posterior Analysis.

3. There are basically two ways to update probabilities of failure. One is by updating the basic random variables and repeating the reliability analysis for re-determining $P_{f}$. The other makes use of the definition of conditional probability and the correlation between the historically observed event and the future event to be assessed. Both lead to the same answers. However, for practical application, especially for high-dimensional problems, the second option is computationally less demanding, hence recommended.

4. The examples demonstrate that the decrease of probability of failure can be orders of magnitude.

5. Survival information may be taken into account for decision-making in inspection and maintenance or in design for newly built structures. In both cases, using the information leads to cost-savings either in terms of lower construction cost or in terms of lower risk. The degree of the decrease in probability of failure and, therefore, in cost is problem-dependent.

6. Notice that so far all calculations have been carried out mainly by means of numerical integration techniques. The last case is a four-dimensional problem and requires already considerable calculation effort and computer memory. Higher dimensional problems might require the use of alternative techniques, such as Markov Chain Monte Carlo sampling.

\section{ACKNOWLEDGMENTS}

The author wishes to gratefully acknowledge the financial support by his employer Deltares.

\section{REFERENCES}

Bayes, T. (1763). "An Essay towards solving a Problem in the Doctrine of Chances." Philosophical Transactions of the Royal Society of London 53: 370-418.

Bligh, W. G. (1910). "Dams, barrages and weirs on porous foundations." Eng. News 64(Dec.): 708.

Ditlevsen, O. (1996). Structural Reliability Methods. Chichester, UK.

Faber, M. H., M. A. Maes, et al. (2007). Principles of risk assessment of engineered systems. 10th International Conference on Application of Statistic and Probability in Civil Engineering (ICASP10).

Hasofer, A. M. and N. C. Lind (1974). "An Exact and Invariant First-Order Reliability Format." ASCE 100(EM1): 111-121.

Hohenbichler, M. and R. Rackwitz (1983). "First order concepts in system reliability." Structural Safety 1: $177-188$.

Rijkswaterstaat (2005). Flood Risks and Safety in the Netherlands (Floris).

Rijkswaterstaat (2007). Hydraulische randvoorwaarden primaire waterkeringen.

Rijkswaterstaat (2008). Decimeringshoogten TMR2006 (RW1708-1_008).

Sellmeijer, J. B. (1988). On the Mechanism of Piping under Impervious Structures. Delft, The Netherlands, Delft University of Technology

Steenbergen, H. M. G. M. and A. C. W. M. Vrouwenvelder (2003). Theoriehandleiding PC-Ring - Deel A: Mechanismebeschrijvingen (2003-CI-R0020). 